\section{AB0761 HISTOPATHOLOGY OF CHECKPOINT INHIBITORS INDUCED ARTHRITIS: RETROSPECTIVE ANALYSIS OF 3 CASES}

M. D. B. Laurent ${ }^{1,2}$, A. Sandrone ${ }^{3}$, D. R. Silvana ${ }^{2}$, C. Frank $^{4}$, P. Renaud ${ }^{5}$, C. Galant ${ }^{6}$, B. Lauwerys ${ }^{7}$, P. Durez ${ }^{7} .{ }^{1}$ Cliniques Universitaires Saint-Luc, Rheumatology, Brussels, Belgium; ${ }^{2} \mathrm{CHU}$ Saint-Pierre, Rheumatology, Brussels, Belgium; ${ }^{3}$ UZ Brussel, Oncology, Brussels, Belgium; ${ }^{4}$ Cliniques Universitaires Saint-Luc, Oncology, Brussels, Belgium; ${ }^{5}$ Clinique Saint-Pierre, Oncology, Ottignies, Belgium; ${ }^{6}$ Cliniques Universitaires Saint-Luc, Pathology, Brussels, Belgium; ${ }^{7}$ Cliniques Universitaires Saint-Luc, Rhumatologie \& Institut de Recherche Expérimentale et Clinique, Brussels, Belgium

Background: Induced arthritis (IA) is a rare immune related adverse event occurring in 0.5 to $2 \%$ of patients exposed to Immune (irAEs) Checkpoint Inhibitors (CPI). In some patients, this condition may be severe and long lasting even after CPI cessation. Cross-reactivity between tumor- and self-antigens was considered in the etiology of several cases of irAEs but disease mechanisms in IA remain elusive.

We systematically collect synovial tissue of patients with IA (Ethical Committee B403201942386).

Objectives: Here, we report the histopathological findings from ultrasound guided (USG) synovial biopsies from the 3 first patients of our cohort. The immune cell infiltration in CPI IA patients is compared to the histopathological findings in rheumatoid arthritis.

Methods: Patients were referred through their oncologist after the onset of an arthritis in the context of a CPI therapy. The diagnostic of arthritis was confirmed by a rheumatologist and by ultrasound assessment. USG biopsies were performed before initiation of specific therapies.

Pathological and immunohistochemical analyses include semi quantitative scoring of the following: synovial hyperplasia, fibrinoid necrosis, chorion cellular infiltrate, vascular hyperplasia, CD3-, CD20-, CD68- and CD138-positive cells. All patients gave written informed consent.

Results: Three patients went through the USG biopsy procedure: 2 males and 1 female. No adverse events were recorded.

All patients had metastatic neoplasms and time-to-arthritis was on average 6.7 months after CPI initiation. The USG biopsies were performed from 1 week after the onset of the IA to 16 months. The target joints displayed strong synovial thickening on ultrasound. Histological and immunohistochemistry studies showed light to moderate synovial hyperplasia and inflammatory cell infiltration of the sublining by macrophages and T cells, but no or little B cells (Table 1).

Table 1. Clinical Characteristics and histopathological analyses on synovial biopsies.

\begin{tabular}{|c|c|c|c|}
\hline & Patient 1 & Patient 2 & Patient 3 \\
\hline Cancer & uterus & bladder & lungs \\
\hline Biopsied joint & first metatarsophalangial joint & wrist & knee \\
\hline Time-to-synovial biopsy & 1 week & 2 months & 16 months \\
\hline $\begin{array}{l}\text { Grey scale on ultrasound } \\
\text { Hematoxylin-eosin staining: }\end{array}$ & $3 / 3$ & $3 / 3$ & $3 / 3$ \\
\hline synovial hyperplasia & + & + & ++ \\
\hline fibrinoid necrosis & 0 & + & ++ \\
\hline chorion cellular infiltrate & + & ++ & + \\
\hline $\begin{array}{l}\text { vascular hyperplasia } \\
\text { Immunochemistry staining: }\end{array}$ & 0 & $0 /+$ & ++ \\
\hline CD3 & + & ++ & + \\
\hline CD20 & 0 & 0 & $0 /+$ \\
\hline CD68 & ++ & $0 /+$ & ++ \\
\hline CD138 & 0 & 0 & $0 /+$ \\
\hline
\end{tabular}

Conclusion: USG synovial biopsies have been safely performed and the histopathological analysis have confirmed the synovitis with mainly a macrophage infiltrate (myeloid pattern). IA synovitis is characterized by synovial hyperplasia and macrophage and $\mathrm{T}$ cell infiltration of the sublining. Increased collaboration between oncologists and rheumatologists is needed to create opportunities for referral of patients between the two specialties and optimize treatment. Further analyses (such as global or single-cell RNA sequencing) are needed to learn more about IA physiopathology.

REFERENCES:

[1] Humby F, Lewis M, Ramamoorthi N \& al. Synovial cellular and molecular signatures stratify clinical response to csDMARD therapy and predict radiographic progression in early rheumatoid arthritis patients. Ann Rheum Dis. 2019 Jun;78(6):761-772. doi: 10.1136/annrheumdis-2018-214539.

[2] Postow MA, Sidlow R, Hellmann MD. Immune-Related Adverse Events Associated with Immune Checkpoint Blockade. N Engl J Med. 2018 Jan 11;378(2):158-168. doi: 10.1056/NEJMra1703481. PMID: 29320654.

Disclosure of Interests: Meric de Bellefon Laurent: None declared, Aspeslagh Sandrone: None declared, Di Romana Silvana: None declared, Cornelis Frank:
None declared, Poncin Renaud: None declared, Christine Galant: None declared, Bernard Lauwerys Employee of: Bernard Lauwerys is currently employed at UCB Biopharma, Patrick Durez: None declared

DOI: 10.1136/annrheumdis-2021-eular.1112

\section{AB0762 THE CHARACTERISTICS OF PERIPHERAL LYMPHOCYTE SUBSETS IN PATIENTS WITH IGG4- RELATED DISEASE}

Y.Y. Wang ${ }^{1}$, X. Yu ${ }^{1}$, R. Su ${ }^{1}$, X. LI ${ }^{1}$, C. Wang ${ }^{1} .{ }^{1}$ The Second Hospital of Shanxi Medical University, Department of Rheumatic Immunology, Taiyuan, China

Background: Immunoglobulin G4-related disease (IgG4-RD) is an autoimmune disease with chronic systemic inflammation and fibrosis. The main feature of the disease was diffuse swell of the affected organs, and the serum IgG4 level was increased. Histopathology of the lesions showed infiltration of IgG4+ plasma cells. However, the pathogenesis of IgG4-RD is still unclear ${ }^{[1]}$.

Objectives: To explore the clinical characteristic of lymphocyte subsets of IgG4-related disease patients, and make comparisons with healthy controls

Methods: A total of 31 patients with IgG4-RD who were admitted to the Rheumatic Immunology Department of the Second Hospital of Shanxi Medical University from January 2016 to June 2020 were included. We collected their Clinical and laboratory data, and selected 30 age and sex matched healthy people as the control group. Flow cytometry was used to detect the percentage and absolute number of lymphocyte subsets ( $T, B, N K, C D 4+T, C D 8+T$ ) and $C D 4+T$ subsets (Th1, Th2, Th17, CD4+CD25+Foxp3+Treg) in peripheral blood of IgG4-RD patients and healthy controls.

Results: (1)The percentage of CD4+T cells in peripheral blood of IgG4-RD patients was higher than that of healthy controls [45.00(33.97-51.48) vs 39.36(33.78-43.30), $\mathrm{P}<0.05]$. (2)The percentage and absolute number of Th17 cells was increased in IgG4-RD patients [1.13(0.70-1.55) vs. 0.77(0.43-1.07), $\mathrm{P}<0.05 ; 7.90(5.20-12.23)$ vs. $5.60(3.12-8.47), P<0.05]$, while the percentage of Treg cells was decreased [3.37(2.82-5.65) vs. 4.96(4.18-6.34), $P<0.01]$. But Treg cells number showed no difference between the two groups. (3) Th17/Treg ratio was significantly increased in IgG4-RD patients $[0.29(0.16-0.46)$ vs. $0.15(0.08$ $0.23), P<0.01$, and it was positively correlated with IgG4-RD response index score $(r=0.491, P<0.01)$. (Table 1$)$.

Table 1. Comparation of absolute number and percentage of peripheral blood lymphocyte subsets between IgG4-RD patients $(n=31)$ and healthy controls $(n=30)$

\begin{tabular}{lccc}
\hline cell subsets & IgG4-RD $(\mathrm{n}=31)$ & $\mathrm{HC}(\mathrm{n}=30)$ & $P$ value \\
\hline $\mathrm{B}$ & $150.59(120.14-212.38)$ & $203.27(152.90-244.27)$ & 0.089 \\
$\mathrm{~B} \%$ & $8.74(6.46-11.45)$ & $10.03(8.26-13.21)$ & 0.059 \\
NK & $261.98(178.82-303.08)$ & $290.83(179.93-451.45)$ & 0.175 \\
NK\% & $13.14(9.92-18.10)$ & $16.50(11.24-21.75)$ & 0.105 \\
CD3+T & $1357.44(992.00-1844.82)$ & $1305.81(978.24-1597.94)$ & 0.708 \\
CD3+T\% & $72.62(69.32-76.96)$ & $71.62(64.97-75.25)$ & 0.135 \\
CD8+T & $436.40(342.71-596.86)$ & $513.50(359.73-620.53)$ & 0.665 \\
CD8+T\% & $24.26(19.48-31.27)$ & $26.50(20.67-32.90)$ & 0.535 \\
CD4+T & $741.00(562.78-1095.52)$ & $664.50(585.52-789.97)$ & 0.428 \\
CD4+T\% & $45.00(33.97-51.48)$ & $39.36(33.78-43.30)$ & 0.032 \\
Th1 & $162.32(108.11-216.61)$ & $144.27(81.52-161.66)$ & 0.094 \\
Th1\% & $19.00(15.24-25.54)$ & $18.46(14.86-24.27)$ & 0.644 \\
Th2 & $7.82(5.35-11.78)$ & $8.25(5.32-10.87)$ & 0.817 \\
Th2\% & $1.00(0.76-1.27)$ & $1.24(0.89-1.64)$ & 0.399 \\
Th17 & $7.90(5.20-12.23)$ & $5.60(3.12-8.47)$ & 0.010 \\
Th17\% & $1.13(0.70-1.55)$ & $0.77(0.43-1.07)$ & 0.026 \\
Treg & $24.45(19.76-44.79)$ & $34.55(27.29-46.57)$ & 0.076 \\
Treg\% & $3.37(2.82-5.65)$ & $4.96(4.18-6.34)$ & 0.003 \\
Th1/Th2 & $20.00(13.78-36.36)$ & $14.97(10.31-21.58)$ & 0.135 \\
Th1/Treg & $5.72(2.92-8.86)$ & $3.68(2.53-4.77)$ & 0.021 \\
Th2/Treg & $0.27(0.16-0.52)$ & $0.22(0.15-0.32)$ & 0.199 \\
Th17/Treg & $0.29(0.16-0.46)$ & $0.15(0.08-0.23)$ & $\mathbf{0 . 0 0 2}$ \\
& & &
\end{tabular}

Conclusion: Th17/Treg immune disorder exists in IgG4-RD patients, and it is related to the disease activity, indicating that Th17/Treg imbalance may be an important pathogenesis of IgG4-RD.

REFERENCES:

[1] Kamisawa T, Zen Y, Pillai S, et al. IgG4-related disease[J]. Lancet, 2015 , 385(9976): 1460-1471.

Disclosure of Interests: None declared

DOI: 10.1136/annrheumdis-2021-eular.1239

\section{AB0763 IGG4-RELATED CORONARY PERIARTERITIS: SYSTEMATIC LITERATURE REVIEW WITH OUR CASE SERIES}

T. Ito ${ }^{1,2}$, S. Fukuil ${ }^{1}$ T. Kanie ${ }^{3}$, T. Nakai ${ }^{1}$, G. Kidoguchi ${ }^{1}$, H. Ozawa ${ }^{1}$, S. Kawaai ${ }^{1}$, Y. Ikeda ${ }^{1}$, A. Koido ${ }^{1}$, Y. Haji ${ }^{2}$, A. Nomura ${ }^{1}$, H. Tamaki ${ }^{1}$, K. Yamaguchi ${ }^{1}$, 
M. Okada'. ' St. Luke's International Hospital, Immuno-Rheumatology Center, Tokyo, Japan; ${ }^{2}$ Daido Hospital, Rheumatology Department, Nagoya, Japan; ${ }^{3}$ St. Luke's International Hospital, Cardiology Department, Tokyo, Japan

Background: Coronary periarteritis is one of the clinical manifestations of IgG4-related disease. It can cause serious conditions such as angina and ruptured aneurysms. Therefore, it is important to recognize the clinical and radiological characteristics, which was little known.

Objectives: We report four patients with IgG4-related coronary periarteritis with a systematic literature review.

Methods: We identified four patients with IgG4-related coronary periarteritis at the St. Luke's International Hospital in Tokyo, Japan from 2014 to 2020. A systematic literature review was conducted for English articles on IgG4-related coronary periarteritis cases with a full text or abstract available. We summarized patient demographics, IgG and IgG4 titers, the site and morphological type of coronary lesion, and other organ involvements.

Results: Our 4 cases and 38 cases identified by the literature review were assessed. Coronary artery lesions were detected by a coronary CT in all but two cases. Wall thickening was the most common type of the lesion. Moreover, there were $32(76.1 \%)$ patients with other organ involvements. The commonest other lesion was peri-aortitis in 21 (50.0\%) patients. In cases with peri-aortitis, IgG and IgG4 titers were significantly higher than those without peri-aortitis (IgG4; 1540 [705.0, 2570.0] vs 246.0 [160.0, 536.3]; $p=0.001$, IgG; 3596.5 [2838.3, 4260.0] vs 1779.0 [1288.3, 1992.8]; $p=0.040)$. In addition, $15(71.4 \%)$ patients of them had three or more IgG4 related organ involvements.

Conclusion: Coronary CT was a useful imaging modality for the diagnosis of IgG4-related coronary periarteritis, and wall thickening was the most common lesion. Moreover, about half cases coexisted with peri-aortitis. Peri-aortitis and other organ involvements should be screened in those with higher IgG and IgG4.

Table 1. Characteristics of our cases and the literature review cases.

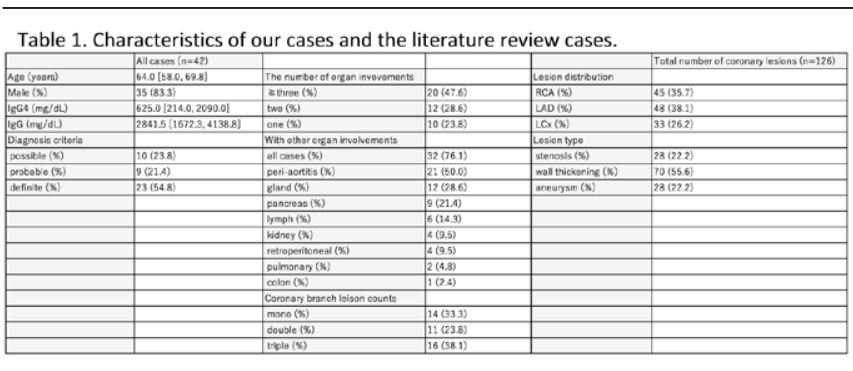

RCA: right coronary artery, LAD: left anterior descending artery, LCx: left circumflex artery

Disclosure of Interests: None declared

DOI: 10.1136/annrheumdis-2021-eular.1293

\section{AB0764 MANIFESTATIONS OF BEHCET'S DISEASE}

R. Goloeva ${ }^{1}$, Z. Alekberova2. ${ }^{1}$ V.A. Nasonova Research Institute of Rheumatology, Systemic Rheumatic Disease, Москва, Russian Federation; ${ }^{1}$ V.A. Nasonova Research Institute of Rheumatology, Systemic Rheumatic Disease, Москва, Russian Federation

Background: Behcet's disease (BD) is a systemic vasculitis of unknown etiology, characterized by recurrent oral and genital erosions and ulcerations, as well as involvement of joints, ocular, vascular, gastrointestinal (GI), and central nervous system (CNS).

Objectives: To study the influence of such factors as patient's gender, age at the disease onset and initial manifestations of the disease on the severity of BD. Methods: 95 patients with confirmed BB were examined. The majority of patients were males (70 men, 25 women) with the $2.8: 1 \mathrm{M}: \mathrm{W}$ ratio. Patients' mean age (median [interquartile range]) was 29 years [22; 34], from 16 to 51 years, the average age at disease onset was 20 years [14; 24]; and mean disease duration was 8 years $[4 ; 15]$

Results: The following clinical manifestations of BD were documented at disease onset (\%): recurrent aphthous stomatitis - 63.1; skin lesions - 12.6; genital ulcers - 4.2; ocular lesions - 6.3; joint syndrome -7.4; CNS lesions -2.1 ; gastrointestinal tract involvement -2.1 ; thrombosis -1 ; epididymitis -1 . $11.6 \%$ of all patients had signs of organ system involvement by the time of establishing BD diagnosis. Recurrent aphthous stomatitis was more common in female patients at $\mathrm{BD}$ onset- $76 \%$ vs. $58.6 \%, p=0.01$, while organ system involvement was more common in males- $14 \%$ vs. $4 \%$ in females, $p=0.02$.
Progression from initial symptoms to the mature BD phenotype allowing to verify the diagnosis occurred within 4.3 years on average $[1 ; 6]$, although $30.5 \%$ of patients progressed to a full-blown disease within 1 year.

Analysis of potential predisposing or precipitating factors prior to BD onset identified such in only 12 patients, including frequent episodes of tonsilitis in 3 patients cold exposure with hypothermia in 2 , dental procedures in 2 , stressful events - in 2 , and one case of each - herpes labialis, frontal sinus puncture and appendectomy. Other patients could not recall any potential precipitator of BD.

Mean age at BD onset was 20 years [14; 24], including $30.5 \%$ of patients with disease onset before 16 years, $22.1 \%$ aged from 16 to 21 years, 36.8\% aged from 21 to 30 years at disease onset, and in remaining $10.5 \%$ the disease manifested after 30 years of age. BD onset in men and women was documented at approximately the same time. There were no signs or correlations indicating that patient's age at disease onset could be associated with specific clinical symptoms in males or females. However, analysis of gender-associated differences in disease severity based on Krause's Clinical Severity Scoring yielded the following results: mild BD was found in $15.7 \%$ men and $44 \%$ women $(p=0.009)$, moderate disease - in $7.1 \%$ men and $16 \%$ women, ( $p>0.05)$, and severe BD - in $77 \%$ men and $40 \%$ women, respectively, $(p=0.001)$. The risk of developing severe BD was 5 -fold higher in men vs women [OR $=5.06,95 \% \mathrm{Cl} 1.7-15.1]$.

Conclusion: First BD symptoms manifested in the majority of patients before the age of 30 years, with only $10 \%$ of patients with BD onset at the age of $>30$ years. In more than $50 \%$ of all patients aphthous stomatitis was the first manifestation of the disease, and was more common in women. Prognostically unfavorable BD signs were found in $11.6 \%$ of patients already at the initial stages of the disease in men. No correlation was found between the age at BD onset and clinical symptoms of the disease in both males and females.

Disclosure of Interests: None declared

DOI: 10.1136/annrheumdis-2021-eular.1364

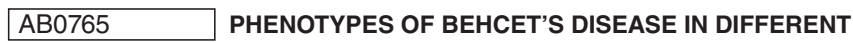 ETHNIC GROUPS}

R. Goloeva ${ }^{1}$, Z. Alekberova2. ${ }^{1}$ V.A. Nasonova Research Institute of Rheumatology, Systemic Rheumatic Disease, Москва, Russian Federation;

${ }^{1}$ V.A. Nasonova Research Institute of Rheumatology, Systemic Rheumatic Disease, Москва, Russian Federation

Background: Early diagnosis and identification of predominant organ system involvement in Behcet's disease (BD) is crucial for identifying most optimal treatment. The study evaluates 6 described phenotypes of BD - cutaneous-mucous, articular, ophthalmic, vascular, neurological and intestinal in different ethnic groups.

Objectives: To assess the occurrence of Behcet's disease (BD) phenotypes in different ethnic groups.

Methods: The study included 202 patients with BD from the 5 most common ethnic groups.

The male-female ratio was 2.4:1. Patients' mean age was 31 years [24;37], mean age at the disease onset was 21 years [15;28]; and mean disease duration was 7 years [3;14]. The severity of BD (mild, moderate and severe) was assessed based on the I. Krause's Clinical Severity Scoring for BD.

Results: Severe BD was more often diagnosed in Azerbaijanis and indigenous residents of Dagestan compared to Russians (75 and $70.4 \%$ vs. $36.2 \%$ ), in Armenians - $50 \%$ and Chechens $-54.5 \%$ out of all BD cases.

Russians were significantly more likely to have a neurological phenotype $(15.5 \%$ vs. $0-9.4 \%$ in all other ethnic groups) and intestinal phenotype (36.2\% vs. 13.8 22.7 in all other ethnic groups). Azerbaijanis demonstrated higher prevalence of ocular involvement $(68.7 \%$ versus $36.2 \%$ in Russians, $50 \%$ - in Chechens and Armenians, and $57 \%$ - in Dagestanis). Dagestanis were more likely to have a vascular phenotype $(40.7 \%$ versus $15.6 \%$ in Azerbaijanis and $18.9 \%$ in Russians). The male/female ratio among Russian patients was 1:1, among Dagestanis 4.4:1, Azerbaijanis 3.5:1, Chechens and Armenians 2.6: 1.

Conclusion: BD phenotypes vary and demonstrate significant association with the patient's ethnic affiliation therefore, ethnicity should be viewed as the prognostic marker of specific organ-system involvement in case of a disease.

Disclosure of Interests: None declared

DOI: 10.1136/annrheumdis-2021-eular.1373

\begin{tabular}{|l|l|}
\hline AB0766 & SUCCESSFUL TEATMENT OF ANKYLOSING \\
SPONDILITIS ASSOCIATED AA AMYLOIDOSIS WITH \\
SECUKINUMAB: A CASE SERIES WITH THREE \\
PATIENTS
\end{tabular}

M. Bektaş ${ }^{1}$, N. Koca ${ }^{1}$, E. Oguz ${ }^{1}$, C. Cetin ${ }^{1}$, B. Ince ${ }^{1}$, Y. Yalçınkaya ${ }^{1}$, B. ArtimEsen ${ }^{1}$, M. L. Ocal ${ }^{1}$, M. Inanc ${ }^{1}$, A. Gül ${ }^{1} .{ }^{1}$ Istanbul Faculty of Medicine, Internal Medicine, Rheumatology Division, Istanbul, Turkey 